\title{
Mechanistic Insights to Pro-Arrhythmogenesis of Short-QT Syndrome Associated with KCNQ1 Gene Mutation
}

\author{
H Zhang ${ }^{1}$, S Kharche ${ }^{1}$, P Stewart ${ }^{1}$, JC Hancox ${ }^{2}$ \\ ${ }^{1}$ The University of Manchester, Manchester, UK \\ ${ }^{2}$ University of Bristol, Bristol, UK
}

\begin{abstract}
Idiopathic short QT (SQT) syndrome is a recently identified, genetically heterogeneous condition characterized by abbreviated QT intervals and an increased susceptibility to arrhythmia and sudden death. In this computer simulation study, we identify the mechanisms by which cellular electrophysiological changes in the SQT syndrome associated with V307L KvLQT1 (KCNQ1) gene mutation (the gene coding the slowly activated rectifier potassium channel current $\left(I_{K s}\right)$ ) increase arrhythmia-risk in human ventricle. Our simulations have shown that V307L gene mutation increases transmural heterogeneity of ventricular repolarisation that leads to an increased tissue's vulnerability for uni-directional conduction block favorable to re-entrant arrhythmia. It also increases tissue's susceptibility to sustain re-entry. This study provided novel mechanistic insights toward understandings of pro-arrhythmogenesis in patients with SQT syndrome subjected to V307L gene mutation.
\end{abstract}

\section{Introduction}

Disorders of ventricular repolarization leading to QT interval shortening have been identified recently in some patients [1-7]. The major features of the 'short QT syndrome' (SQTS) are characterized by shortened QT intervals in ECG and sudden cardiac death and arrhythmic events in the absence of structural heart disease [1-2]. So far, three genetic variants of the SQTS have been identified, which include mutations to $h E R G$ (SQT1), KCNQ1 (the gene responsible for the (KvLQT1) $\alpha$-subunit of the $\mathrm{I}_{\mathrm{Ks}}$ channel) [5-6] and KCNJ2 (SQT3) [7]. Although changes to $\mathrm{I}_{\mathrm{Kr}}, \mathrm{I}_{\mathrm{Ks}}$ and $\mathrm{I}_{\mathrm{K} 1}$ associated with these mutations are anticipated to shorten action potential duration (APD) and, thereby, refractoriness [4-5, 7], the precise mechanism(s) leading to increased arrhythmic risk in the SQTS are not known.

In this study, we used computer simulations to define arrhythmogenic mechanisms in the SQT2 variant, in which specific changes to ionic current kinetics of the slowly activated potassium channel $\left(\mathrm{I}_{\mathrm{Ks}}\right)$ current induced by the V307L KCNQ1 mutation [5] were incorporated into a biophysically detailed computer model of human ventricle. Using the model, we determined the effects of the V307L KCNQ1 mutation on transmural heterogeneity of repolarization, the vulnerability of tissue to unidirectional conduction block and the susceptibility of the tissue to sustain re-entry. The results obtained explain how susceptibility to re-entrant arrhythmia may occur in this form of the SQTS.

\section{Methods}

Model of single cell and $\mathrm{I}_{\mathrm{Ks}}$ channel kinetics. The human ventricular tissue model recently developed by ten Tusscher et al. [8] was used. Equations of $\mathrm{I}_{\mathrm{Ks}}$ in the model were modified to incorporate experimental data of Bellocq et al. [5] on KvLQT1 V307L mutation-induced changes in $\mathrm{I}_{\mathrm{Ks}}$ channel kinetics that include halfactivation voltage $\left(\mathrm{V}_{0.5}\right)$, slope factor and activation rate. Four different cases were considered in respect of the KvLQT1 V307L mutation-induced changes on the steady state of activation and activation rate. These are detailed in Table 1. To simulate the transmural heterogeneity in the ventricular APs across the human ventricle wall, different ionic channel current densities for $I_{\text {to }}$ and $I_{K s}$ were used for endocardial (endo-), mid-myocardial (M-) and epicardial (epi-) ventricular myocytes [8].

Multicellular transmural ventricular tissue model. A transmural ventricular tissue model was constructed using the equation:

$$
\frac{\partial V_{m}}{\partial t}=-I_{t o t}+\nabla \bullet\left(D \nabla V_{m}\right)
$$

where $\mathrm{D}$ is the diffusion parameter modelling the intercellular electrical coupling via gap junctions. $\mathrm{I}_{\text {tot }}$ is the total ionic current flowing across the cell membrane and is modelled by the ten Tusscher et al. equations [8]. In the model heterogeneous electrical properties of cells are incorporated by varying the maximal ionic channel 
Table 1. KvLQT1 V307L mutation-induced changes in $\mathrm{I}_{\mathrm{Ks}}$ channel kinetics.

\begin{tabular}{|c|c|c|c|c|}
\hline & $\begin{array}{c}\mathrm{a} \\
(\mathrm{WT})\end{array}$ & $\begin{array}{c}\mathrm{b} \\
(\text { Het })\end{array}$ & $\begin{array}{c}\mathrm{c} \\
(\text { Hom })\end{array}$ & $\begin{array}{c}\mathrm{D} \\
\text { (HomIsKred) }\end{array}$ \\
\hline $\begin{array}{c}\mathrm{V}_{0.5} \\
(\mathrm{mV})\end{array}$ & -5.9 & -20.6 & -24.0 & -24.0 \\
\hline $\begin{array}{c}\mathrm{S} \\
(\mathrm{mV})\end{array}$ & 17.4 & 10.9 & 16.0 & 16.0 \\
\hline $\begin{array}{c}\text { Scale } \\
\text { of } \\
\text { activatio } \\
\text { n time- } \\
\text { constant }\end{array}$ & 1 & 0.7 & 0.52 & 0.32 \\
\hline
\end{tabular}

conductance of various currents for the endo-, M- and epi- cells. In simulations, D was set to a constant value of $0.45 \mathrm{~cm}^{2} \mathrm{~s}^{-1}$ that gave a conduction velocity of a planar wave across the ventricular wall at $0.4 \mathrm{~m} \mathrm{~s}^{-1}$. D was set to be homogeneous throughout the strand, except for a 5fold decrease at the epi-M border as suggested by Gima and Rudy [9].

Measurement of transmural heterogeneity in ventricular repolarisation. Transmural heterogeneity in ventricular repolarisation was measured by the spatial gradient of action potential duration at $90 \%$ repolarisation $\left(\mathrm{APD}_{90}\right)$ of all cells along the 1D strand. AP was first initiated at the endo- end of the strand by a series of 10 suprathreshold stimuli as used by ten Tusscher et al. [8]. The time interval between successive stimuli was $500 \mathrm{~ms}$. The evoked AP propagated from the endo- region towards M- and then the epi- regions. APs elicited by the fifth stimulus in the series were used to compute the spatial distribution of $\mathrm{APD}_{90}$ and its spatial derivative along the 1D strand.

Computing the pseudo-ECG. The pseudo-ECG was computed following the method of Gima and Rudy [9].

Measurement of Temporal vulnerability. The temporal vulnerability of cardiac tissue to re-entry was quantified by the width of a time window during which a test stimulus applied to the refractory tail of a previous excitation wave in the 1D tissue model evoked unidirectional conduction [10]. A series of 5 conditioning waves were initiated by supra-threshold stimuli (with strength and durations as stated above for AP simulations) applied to a $0.6 \mathrm{~mm}$ segment at the endoend. A test stimulus (with the same size, strength and duration as the one used for a conditioning wave) was applied at the epi- part with $1.05 \mathrm{~mm}$ distance to the Mepi junction. A unidirectional block falls in a time window (T1, T2), whose width $(\mathrm{T} 2-\mathrm{T} 1)$ provides a measure of the vulnerability of the tissue to re-entry. This was measured for normal and the various KvLQT1 V307L mutation expression conditions.

Initiation of re-entry and measurement of spatial vulnerability. Re-entry in a 2D tissue model was initiated by the standard S1-S2 protocol. S1 was applied to the endocardial surface of the endo- layer to evoke a planar excitation wave propagating towards the epi- layer. During the vulnerable window, a S2 of stimulus was applied to a local epi- area of the tissue within the vulnerable window to evoke uni-directional propagation that can lead to re-entry. Each of the 2 ends of the S2evoked excitation wave forms a tip of a pair of counterrotating spiral waves. A large size of S2 is required to provide a sufficient re-entrant pathway of the two spiral arms, which is dependent on their wavelength. If the size of S2 is big enough, then the two spiral waves have sufficient circuit substrates and survive. Otherwise, the spiral wave pair collide and terminate. In order to evaluate the critical size of re-entrant pathway of tissue, we estimated the minimal spatial length of S2 that supported the formation of re-entrant spiral waves, under control and KvLQT1 V307L mutation conditions. The minimal length of S2 gives an inverse index of the spatial vulnerability of tissue to re-entry: the larger the minimal length, the harder for initiation of re-entry.

\section{Results}

We first simulated ventricular APs for epi-, M- and endo- cells under WT (a) and under the various KvLQT1 V307L mutant expression conditions (c-d). In all the three cell models, KvLQT1 V307L mutation condition produced an increased and rapidly activated $\mathrm{I}_{\mathrm{Ks}}$, leading to APD abbreviation. However, the APD abbreviation is inhomogeneous - with a larger APD reduction in the endo- and epi- cells than in the $M$ - cells. As a consequence, the $\mathrm{APD}_{90}$ difference between endo-M-epi cells was augmented.

Using a 1D-strand model a pseudo-ECG was computed for the WT (a) and for the three KvLQT1 V307L mutation conditions (b-d). The results are shown in Figure 1. In response to a series of supra-threshold stimuli to the endo-end of the strand, the evoked AP propagated from the endo- end towards the $\mathrm{M}$ - and then the epi-parts of the strand as shown in panel A, which represented a colour coded space-time plot of APs along the strand. From this propagating excitation wave, the pseudo-QT interval was calculated for conditions of (ad). In these simulations the pseudo-QT interval was shortened progressively from $339 \mathrm{~ms}$ in the WT to 285 ms (Het), $254 \mathrm{~ms}$ (Hom) and $216 \mathrm{~ms}$ (HomIsKred) and T wave amplitude increased. $\mathrm{T}$ wave width (measured by the time interval between $T_{\text {peak }}$ and $T_{\text {end }}$ ) also increased from $25 \mathrm{~ms}$ (WT) to $30 \mathrm{~ms}$ (Het), $31 \mathrm{~ms}$ (Hom) and 32 ms (HomIsKred).

The KvLQT1 V307L mutation conditions also augmented $\mathrm{APD}_{90}$ dispersion in the intact $1 \mathrm{D}$ transmural 

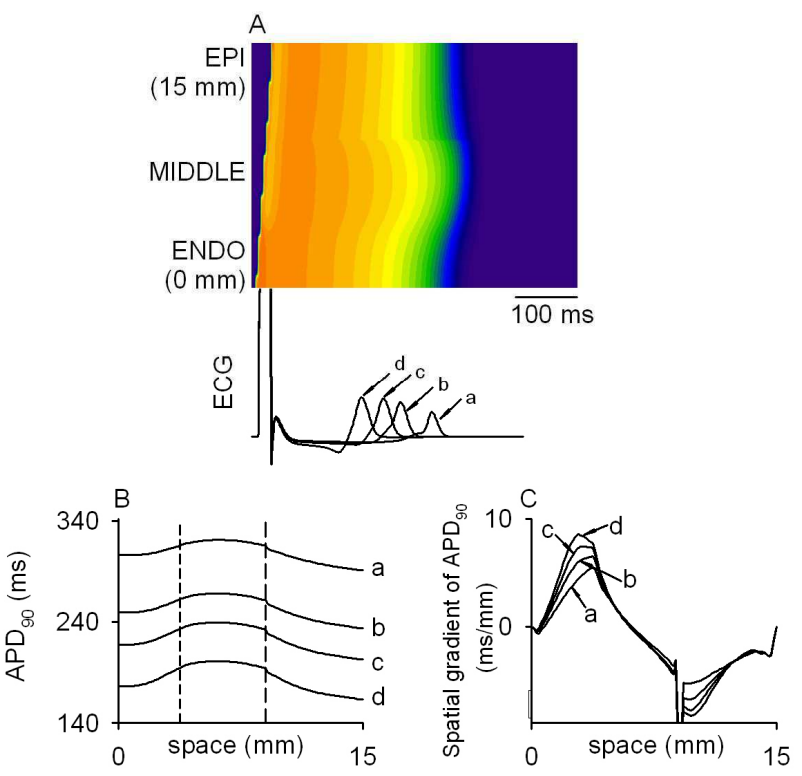

Figure 1. A. Space-time plot of AP propagation and the computed pseudo-ECG for WT (a) and variant V307L KvLQT1 gene mutations. Membrane potential is colour coded from blue for $-100 \mathrm{mV}$ to red for $+50 \mathrm{mV}$. B. Spatial distribution of $\mathrm{APD}_{90}$ on 1D transmural strand in conditions (a)-(d). C. Spatial gradient of $\mathrm{APD}_{90}$ on the strand.

strand as shown in Figure 1B\&C. Figure 1B plots the spatial distribution of $\mathrm{APD}_{90}$ for WT and KvLQT1 V307L mutation conditions. Whilst the measured APD 90 was decreased in all mutation conditions along the whole strand, its spatial gradient was augmented as shown in Figure $1 \mathrm{C}$. In both $1 \mathrm{~B}$ and $1 \mathrm{C}$, a sharp transition of $\mathrm{APD}_{90}$ at the epi-M border is observable; this is due to the discontinuity of intercellular coupling at the border and is consistent with previous experimental observations made from a canine left ventricular wedge preparation [9].

Simulations were then performed to determine whether or not the KvLQT1 V307L mutation could be shown to generate an electrical substrate at the tissue level that would favour increased susceptibility to ventricular arrhythmia. Using 1D-tissue strand simulations we quantified the vulnerability of tissue to unidirectional conduction block in response to a test stimulus applied to the refractory tail of a previous excitation wave for WT and each of the three KvLQT1 V307L mutation expression cases.

Figure 2 shows the $5^{\text {th }}$ conditioning excitation wave and the response of the tissue to a test stimulus applied at the epi- portion of the strand (marked by the arrow) a time delay $\Delta \mathrm{t}$ after the fifth stimulus. In Figure $2 \mathrm{~A}$, the test stimulus was applied sufficiently early $(\Delta \mathrm{t}=360 \mathrm{~ms})$
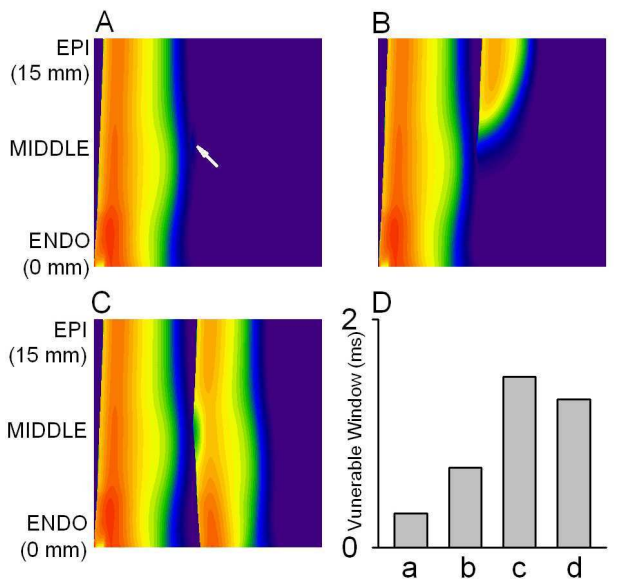

Figure 2. Response of tissue to a premature test stimulus applied at a local epi-part of the tissue (marked by arrow) at various time intervals $(\Delta \mathrm{t})$ after the fifth conditioning wave. A. $\Delta \mathrm{t}=360 \mathrm{~ms}$. Bidirectional block. B. $\Delta \mathrm{t}=361.9$ $\mathrm{ms}$, unidirectional conduction block. C. $\Delta \mathrm{t}=365 \mathrm{~ms}$, bidirectional conduction. D. Vulnerability of tissue in WT (a) and 307L KvLQT1 gene mutation conditions (b-d).

after the previous excitation that the tissue surrounding the stimulus site did not have time to recover enough for re-excitation to occur. As a consequence, a bi-directional conduction block was observed. In Figure 2B, the test stimulus was applied within the vulnerable window $(\Delta \mathrm{t}=$ $361.9 \mathrm{~ms}$ ) that produced a unidirectional block. In twodimensions, unidirectional conduction block is an essential factor to generate re-entrant excitation. In Figure $2 \mathrm{C}$, the test stimulus was applied well after the tissue surrounding the stimulus site (in both anterograde and retrograde directions) had enough time to recover from the previous excitation $(\Delta \mathrm{t}=365 \mathrm{~ms})$ and, consequently, bi-directional conduction was observed. Figure 2D shows the width of the vulnerable time window, during which a test stimulus applied at the epi-portion of the tissue strand resulted in unidirectional block under WT and different KvLQT1 V307L mutant expression conditions. The computed vulnerable window increased from $0.3 \mathrm{~ms}$ in the WT to $0.7 \mathrm{~ms}, 1.5 \mathrm{~ms}$ and $1.3 \mathrm{~ms}$ under Het, Hom, HomIsKred condition respectively. Thus the KvLQT1 V307L mutation increased tissue's vulnerability to unidirectional conduction block by over $230-500 \%$.

A premature test stimulus during the vulnerable window in $2 \mathrm{D}$ tissue produced uni-directional conduction leading to genesis of re-entry (spiral wave) as shown in Figure 3. In Figure 3A, the length of the S2 stimulus was $120 \mathrm{~mm}$. The S2 evoked excitation wave propagated unidirectionally and formed a pair of spiral waves (Figure $3 \mathrm{~A})$. However, as the size of S2 was small, there was not 
enough room to accommodate the pair and they self-

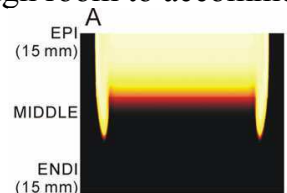
B
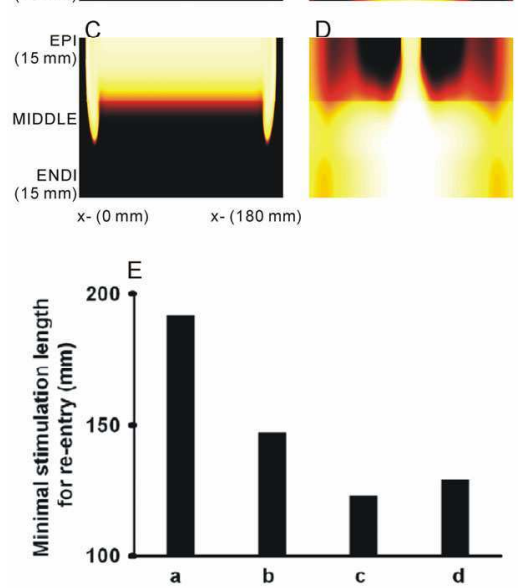

Figure 3. Measurement of critical length of ventriclular re-entrant pathway in KvLQT1 V307L (Hom) condition. A-B. Snapshots of reentrant excitation wave evoked by a S2 stimulus under the critical size. Reentry selfterminated. C-D. Snapshots of reentrant excitation wave evoked by a S2 stimulus above the critical size. Reentry sustained. E. Measured critical length of tissue's reentrant pathway for WT (a) and KvLQT1 V307L mutations (b-d).

terminated (Figure 3B). In Figure 3C, the length of the S2 stimulus was $130 \mathrm{~mm}$, just above the critical size (124 $\mathrm{mm})$. In this case, there was sufficiently large room to accommodate them (Figure 3C) and the two spiral waves survived and persisted (Figure 3D). The measured minimal (critical) length (Figure 3E) was $195 \mathrm{~mm}$ for the WT condition (a), $147 \mathrm{~mm}$ for the Het condition (b), 124 $\mathrm{mm}$ for the Hom condition (c) and $130 \mathrm{~mm}$ for HomIsKred condition (d). There was a significant reduction in the critical size necessary to support the formation and maintenance of reentry, reflecting the likely impact of this mutation on stability of the clinical arrhythmia. The dynamical behaviours of the evoked reentrant spiral wave were also affected by the KvLQT1 V307L gene mutation. For the WT condition, the spiral wave spontaneously terminated due to the long effective refractory period. However, as the KvLQT1 V307L mutation shortened the ERP, this would be anticipated to reduce the size of the substrate required to facilitate and maintain re-entry. Consistent with this notion, the initiated spiral wave was found to be sustained under KvLQT1 V307L mutation conditions (Figure 6 B-D), thereby demonstrating an increased susceptibility to arrhythmia.

\section{Discussion and conclusions}

From these simulations it can be concluded that: (i) the KvLQT1 V307L mutation leads to QT interval shortening; (ii) the V307L mutation leads to an increased transmural heterogeneity in ventricular repolarisation that increases tissue's vulnerability to the genesis of re-entry by a premature excitation; (iii) the KvLQT1 V307L mutation shortens tissue's APD that facilitates the maintenance of re-entry. These findings both substantiate a causal link between the KvLQT1 V307L mutation and QT interval shortening, and offer a novel explanation for increased susceptibility to re-entry and perpetuation of reentrant arrhythmia in this form of SQTS.

\section{Acknowledgements}

This work is supported BBSRC (UK, BBS/B/1678X) and BHF (UK, PG/03/140/16236).

\section{References}

[1] Gussak I et al. Idiopathic short QT interval: a new clinical syndrome? Cardiology 2000; 94: 99-102.

[2] Gussak I et al. ECG phenomenon of idiopathic and paradoxical short QT intervals. Cardiac Electrophysiology Review 2002; 6: 49-53.

[3] Gaita F et al. Short QT syndrome: a familial cause of sudden death. Circulation 2003; 108: 965-97

[4] Brugada R et al. Sudden death associated with short-QT syndrome linked to mutations in HERG. Circulation 2004; 109: 30-35.

[5] Bellocq $\mathrm{C}$ et al. Mutation in the KCNQ1 gene leading to the short QT-interval syndrome. Circulation 2004; 109(20):2394-7.

[6] Honk K et al. KCNQ1 mutation responsible for atrial fibrillation and short QT syndrome in utero. Cardiovasc Res. 2005; 68: 432.440.

[7] Priori SG et al. A novel form of short QT syndrome (SQT3) is caused by a mutation in the KCNJ2 gene. Circ Res 2005; 96: 000-000.

[8] ten Tusscher KH et al. Am J Physiol Heart Circ Physiol. 2004; 286(4): H1573-89.

[9] Gima K \& Rudy Y. Ionic current basis of electrocardiographic waveforms: a model study. Circ Res. 2002; 90: 889-896.

[10] Zhang H, Holden AV. One-dimensional modelling of the vulnerability to re-entry of homogeneous atrial tissue. J Theor Biol 1997; 184: 119-124.

Address for correspondence

Dr. Henggui Zhang

Biological Physics Group

School of Physics \& Astronomy

The University of Manchester

Manchester, M13 9PL, UK

E-mail address: henggui.zhang@manchester.ac.uk 